\title{
Charmed mesons with a symmetry-preserving contact interaction
}

\author{
Fernando E. Serna, ${ }^{1}$ Bruno El-Bennich, ${ }^{2}$ and Gastão Krein ${ }^{1}$ \\ ${ }^{1}$ Instituto de Física Teórica, Universidade Estadual Paulista, \\ Rua Dr. Bento Teobaldo Ferraz, 271 - Bloco II, 01140-070 São Paulo, SP, Brazil \\ ${ }^{2}$ Laboratorio de Física Teórica e Computacional, Universidade Cruzeiro do Sul, \\ Rua Galvão Bueno 868, 01506-000 São Paulo SP, Brazil
}

\begin{abstract}
A symmetry-preserving treatment of a vector-vector contact interaction is used to study charmed heavy-light mesons. The contact interaction is a representation of nonperturbative kernels used in Dyson-Schwinger and Bethe-Salpeter equations of QCD. The Dyson-Schwinger equation is solved for the $u, d, s$ and $c$ quark propagators and the bound-state Bethe-Salpeter amplitudes respecting spacetime-translation invariance and the Ward-Green-Takahashi identities associated with global symmetries of QCD are obtained to calculate masses and electroweak decay constants of the pseudoscalar $\pi, K, D$ and $D_{s}$ and vector $\rho, K^{*}, D^{*}$, and $D_{s}^{*}$ mesons. The predictions of the model are in good agreement with available experimental and lattice QCD data.
\end{abstract}

\section{INTRODUCTION}

Heavy-light $Q \bar{q}$ (and $\bar{Q} q$ ) mesons, such as the $B$ and the $D$, are interesting bound states of quantum chromodynamics (QCD). They are interesting because they are composed of quarks belonging to two limiting mass sectors of QCD with associated emergent approximate symmetries: the sector of light quarks $q=(u, d, s)$, with masses $m_{q} \ll \Lambda_{\mathrm{QCD}}$, and the sector of heavy quarks $Q=(c, b, t)$, with masses $m_{Q} \gg \Lambda_{\mathrm{QCD}}$, where $\Lambda_{\mathrm{QCD}}$ is the energy scale at which the theory becomes strongly coupled, thereby implying that the characteristic size of a typical hadron is $\Lambda_{\mathrm{QCD}}^{-1}$. In the $m_{q} \rightarrow 0$ limit, $\mathrm{QCD}$ acquires an $S U(3)_{L} \times S U(3)_{R}$ chiral symmetry that is dynamically broken by the strong QCD interactions to an $S U(3)_{V}$ flavor symmetry; the eight pseudoscalar mesons $\pi^{0}, \pi^{ \pm}, K^{0}, \bar{K}^{0}, K^{ \pm}$, and $\eta$ are identified with the (pseudo-)Goldstone bosons associated with the dynamical breaking of the symmetry. In the $m_{Q} \rightarrow \infty$ limit, the interactions of a heavy quark, regardless of its flavor, within a heavy-light meson become independent of its spin, a feature that gives rise to a spin-flavor heavy quark $U\left(2 N_{h}\right)$ symmetry $-2 N_{h}=2($ spin $) \times N_{h}$ (heavy flavors). Moreover, since the average velocity $v$ of the heavy quark in a $Q \bar{q}$ bound state is changed very little by the interactions, as $\Delta v=\Delta p / m_{Q} \sim \Lambda_{\mathrm{QCD}} / m_{Q} \ll 1$, the light quark dynamics occurs in the background of a strong color field of an essentially static spectator. Therefore, heavy-light mesons offer a unique opportunity to learn about features of dynamical chiral symmetry breaking $(\mathrm{D} \chi \mathrm{SB})$ in a spin- and flavor-independent environment provided by the heavy quark.

In fact, the approximate chiral and heavy quark spinflavor symmetries can be combined to construct powerful effective field theories to make predictions for a wealth of processes involving heavy-light mesons, like electroweak decay rates and their low-energy interactions with other hadrons $[1,2]$. However, as with many other effective field theories, coupling constants in the Lagrangian of chiral heavy-quark effective field theories, and also form factors associated with decay matrix elements, are of nonperturbative origin and need be fixed from data or from other theoretical source, as simulations of QCD on a space-time lattice or calculations using nonperturbative methods in the continuum. The present work is related to the latter; in particular, to an approach based on the Dyson-Schwinger (DS) and Bethe-Salpeter (BS) equations of QCD $[3,4]$.

The DS and BS equations consist of an infinite set of coupled integral equations; once a truncation scheme is specified, they define a tractable and predictive problem. Systematic, symmetry-preserving, nonpertubatively renormalizable truncation schemes, continuously developed since the 1990, reached a high degree of sophistication and have proven very successful in describing and correlating a great variety of phenomena in the light-quark sector of QCD [5, 6]. Symmetry-preserving schemes make use, in an essential way, of the WardGreen-Takahashi (WGT) identities reflecting global symmetries and their explicit breaking; they impose stringent relationships between the interaction kernels entering DS equations for quark and gluon propagators and quarkgluon vertices and those entering BS equations for bound states [7].

Notwithstanding the advances and successes, challenges still remain in describing simultaneously the masses and decay constants of light- and heavy-flavored mesons within a single interaction-truncation scheme [817]; in particular, the disagreements with data for the electroweak decay constants are substantial $[9,17]$. Although one can expect that the challenges will be overcome in a foreseeable future, there is pressing need for different pieces of information on the structure and interactions of such mesons for guiding new experiments at existing and forthcoming facilities, aiming at e.g. production of exotic hadrons like the X,Y,Z hadrons in heavyion collisions and creation of exotic nuclear bound states with charmed hadrons $[18,19]$. Predictions for masses, strong couplings, decay rates, and interaction cross sections are needed as functions of external parameters like temperature, baryon density and magnetic field, delivered in a form that can be used efficiently in transport and hydrodynamic simulation codes of such complex ex- 
periments. Given these circumstances and demands, in the present paper we explore the effectiveness in describing properties of $D$ mesons of a simpler alternative based on a four-fermion contact interaction (CI) model embedded in a symmetry-preserving scheme [20].

Fermionic contact interactions find widespread applications in hadron physics as evidenced by the popular use of models inspired by the Nambu-Jona-Lasinio (NJL) [21]. A great deal of qualitative insight on the phenomenon of hadron mass generation via $\mathrm{D} \chi \mathrm{SB}$ and the role of the $\pi^{0}, \pi^{ \pm}, K^{0}, \bar{K}^{0}, K^{ \pm}$, and $\eta$ mesons as the associated (pseudo-)Goldstone bosons has been gleaned from such models-for reviews, see Refs. [2225]. On the other hand, the lack of confinement and non-renormalizabilty are the major weaknesses of these CI models. The non-renormalizability, notably, carries along the danger of introducing gross violations of global symmetries due to the regularization procedure; ambiguities arising from momentum shifts in divergent integrals and severe dependence of results on choices of momentum sharing between a heavy and a light quark in a bound state are the main causes of the problems.

A new perspective, however, has recently emerged with the implementation by Gutiérrez-Guerrero, Bashir, Cloët and Roberts [26] (GBCR henceforth) of a confining, symmetry-preserving treatment of a vector-vector CI as a simplified ansatz for the gluon's two-point Schwinger function commonly employed in the kernel of the quark's DS equation $[3,12,27,28]$. By introducing a mechanism that ensures the absence of quark production thresholds [29], a feature of a confining theory, and embedding the interaction in a global-symmetry-preserving, rainbow-ladder $(\mathrm{RL})$ truncation framework of the DS and BS equations [30, 31], the GBCR scheme has enhanced the CI's capacity to describe in a unified manner a diverse array of phenomena that include the light-quark meson and baryon spectra as well as their electroweak, elastic and transition form factors [32-40] in appropriate kinematic regimes. Very recently, the scheme has been extended to calculate ground-state masses and weak decay constants of heavy charmonia [41], and also elastic and transition form factors of $\eta_{c}(1 S)$ [42].

In this paper, we examine the GBCR approach within the perspective of a subtraction scheme that allows one to isolate symmetry-violating contributions in BS amplitudes for arbitrary momentum routing in occurring divergent integrals. The scheme has been employed in NJL model calculations in vacuum $[43,44]$ and at finite temperature and baryon density [45-48], and very recently to investigate the critical behavior of quark matter in presence of a chiral imbalance [49]. The scheme is inspired in the method introduced in Ref. [50], the aim of which is the treatment of divergent Feynman integrals without specification of an explicit regulator; it shares similarities [51] with the Bogoliubov, Parasiuk, Hepp, Zimmermann (BPHZ) renormalization subtraction scheme [52], which uses systematic subtractions of momentum space integrals to isolate divergences.
We here take advantage of the strengths of the subtraction scheme and apply it to heavy-light mesons within the CI approach. Heavy-light mesons were studied previously in the NJL model using a traditional cutoff regularization [53]. However, a wrong pattern in the ordering of the pseudoscalar decay constants, $f_{D}<f_{\pi}$ was found - the experimental pattern is $f_{\pi}<f_{K}<f_{D}$. More recently, a similar incorrect ordering in the decay constants was encountered within the CI framework for heavy charmonia in Ref. [41]. In addition, as already mentioned, even implementing a more realistic interaction in the RL truncation of the DS and BS equations, the weak decay constants of heavy-light mesons compare unfavorably [9] with predictions of lattice-QCD and the $D$ to $D_{s}$ mass difference is vanishingly small [14]. Possible causes for the failure of this truncation in heavylight systems have been put forward in Ref. [54]. While not being a substitute for a full-fledged QCD-based DSBS framework currently under intense development, it is nonetheless legitimate to expect that the capacity of a CI scheme in providing useful insight on heavy-light mesons is enhanced when it respects fundamental spacetime and internal symmetries of QCD.

This paper is organized as follows: in Sec. II we summarize the essentials of DSE, BSE and Ward-GreenTakahashi identities (WGTI) and introduce the CI scheme employed herein. In Sec. III, we introduce the subtraction scheme to deal with divergent integrals that occur in the Bethe-Salpeter kernels due to the simplification of the CI and discuss the consequences of the axialvector WGTI for these divergences. In Sec. IV we present our numerical results for masses and weak decay constant of the mesons of interest using the subtraction scheme introduced in Sec. III We discuss our results and compare them with those obtained in Ref. [41]. Finally, in Sec. V we conclude with some final remarks about this work.

\section{DS AND BS EQUATIONS AND WGT IDENTITIES}

We begin with a brief review of the basic elements of the GBCR contact-interaction scheme [26]. We consider the inhomogeneous BS equation for a quark and antiquark state of total momentum $P$ (here and in the following we omit renormalization constants):

$$
\begin{aligned}
{\left[\Gamma_{\mathcal{M}}(k ; P)\right]_{A B}=} & \mathcal{M}_{A B}+\int_{q}[K(k, q ; P)]_{A C, D B} \\
& \times\left[S\left(q_{+}\right) \Gamma_{\mathcal{M}}(q ; P) S\left(q_{-}\right)\right]_{C D},
\end{aligned}
$$

where $\int_{q} \equiv \int d^{4} q /(2 \pi)^{4}, \mathcal{M}$ represents the Dirac spinor structure of the state, $K(q, k ; P)$ is the fully amputated quark-antiquark scattering kernel; $A, B, \cdots$ denote collectively color, flavor, and spinor indices; $q_{ \pm}=q \pm \eta_{ \pm} P$, with $\eta_{+}+\eta_{-}=1$ and $q$ is the relative momentum. $S(k)$ is the dressed-quark propagator given by a DSE; for a 
given flavor $f$ the general form of this DSE is (in Euclidean metric)

$$
\begin{aligned}
S_{f}^{-1}(k)= & i \gamma \cdot k+m_{f} \\
& +\int_{q} g^{2} D_{\mu \nu}(k-q) \frac{\lambda^{a}}{2} \gamma_{\mu} S_{f}(q) \Gamma_{\nu}^{a f}(q, k),
\end{aligned}
$$

where $m_{f}$ is the current-quark mass. In here, we are interested in the flavor-nonsinglet axial-vector $\Gamma_{5 \mu}^{l h}(k ; P)$ and pseudoscalar $\Gamma_{\mathrm{PS}}^{l h}(k ; P)$ amplitudes for a quarkantiquark pair of a light $(l)$ and a heavy $(h)$ quark, with $\mathcal{M}_{5 \mu}=\gamma_{5} \gamma_{\mu}$ and $\mathcal{M}_{P S}=\gamma_{5}$ respectively. Spacetimetranslation invariance requires that no observable can depend on the choice of the momentum routing in quark propagators in Eq. (1); that is, physical results must be independent of $\eta_{ \pm}$.

Associated with $\Gamma_{5 \mu}^{l h}$ is the WGT identity.

$$
\begin{aligned}
P_{\mu} \Gamma_{5 \mu}^{l h}(k ; P)= & S_{l}^{-1}\left(k_{+}\right) i \gamma_{5}+i \gamma_{5} S_{h}^{-1}\left(k_{-}\right) \\
& -i\left(m_{l}+m_{h}\right) \Gamma_{\mathrm{PS}}^{l h}(k ; P),
\end{aligned}
$$

where $\Gamma_{P S}^{l h}(k ; P)$ is the pseudoscalar vertex; both $\Gamma_{5 \mu}^{l h}(k ; P)$ and $\Gamma_{\mathrm{PS}}^{l h}(k ; P)$ obey inhomogeneous BS equation (1). Pseudoscalar meson bound states are obtained from the solution of the homogeneous equation for $\Gamma_{\mathrm{PS}}^{l h}(k ; P)$

$$
\begin{aligned}
{\left[\Gamma_{\mathrm{PS}}^{l h}(k ; P)\right]_{A B}=} & \int_{q}\left[K^{l h}(k, q ; P)\right]_{A C, D B} \\
& \times\left[S_{l}\left(q_{+}\right) \Gamma_{\mathrm{PS}}^{l h}(q ; P) S_{h}\left(q_{-}\right)\right]_{C D},
\end{aligned}
$$

where here $A, B, \cdots$ denote color and spinor indices only. The general form of the of $\Gamma_{\mathrm{PS}}^{l h}(k ; P)$ is

$$
\begin{aligned}
\Gamma_{\mathrm{PS}}^{l h}(k, P)= & \gamma_{5}\left[i E_{\mathrm{PS}}^{l h}+\gamma \cdot P F_{\mathrm{PS}}^{l h}+\gamma \cdot k G_{\mathrm{PS}}^{l h}\right. \\
& \left.+\sigma_{\mu \nu} k_{\mu} P_{\nu} H_{\mathrm{PS}}^{l h}\right],
\end{aligned}
$$

where $E_{\mathrm{PS}}^{l h}, F_{\mathrm{PS}}^{l h}, \cdots$ are functions of $k, P$ and $k \cdot P$. The meson mass, $m_{\mathrm{PS}}$, is the eigenvalue for the value $P^{2}=$ $-m_{\mathrm{PS}}^{2}$ that solves Eq. (4).

The CI scheme introduced in Ref. [26] amounts to the following replacement in Eq. (2)

$$
\begin{aligned}
g D_{\mu \nu}(k-q) g \Gamma_{\nu}^{a f}(q, k) & \rightarrow\left(\frac{4 \pi \alpha_{\mathrm{IR}}}{m_{g}^{2}}\right)^{f} \frac{\lambda^{a}}{2} \gamma_{\mu} \\
& \equiv\left(\frac{1}{m_{\mathrm{G}}^{f}}\right)^{2} \frac{\lambda^{a}}{2} \gamma_{\mu},
\end{aligned}
$$

where $m_{g}$ is a gluon mass-scale and $\alpha_{\mathrm{IR}}$ is a coupling strength parameter. Note that a flavor dependence in the interaction strength is due to the flavor dependence of the full quark-gluon vertex $\Gamma_{\mu}^{a f}$. The flavor dependence is important to accommodate the fact that heavy-flavor quarks probe shorter distances than light-flavor quarks at the corresponding quark-gluon vertices, thereby implying a smaller coupling strength for heavy-flavor quarks [54]. This fact was used previously in the NJL model to mimic the short-distance and weak-coupling physics in quark matter at high temperatures and baryon densities [46, $55]$. Very recently, Refs. [41, 42] have shown that good agreement with experiment for heavy-charmonia masses can be obtained by using a weaker coupling strength and a simultaneous increase in the ultraviolet cutoff of the CI of Ref. [26]. We anticipate that the same turns out to be relevant for heavy-light mesons and write for the BS kernel

$$
\left[K^{l h}(k, q ; P)\right]_{A C, D B}=-\frac{1}{m_{\mathrm{G}}^{l} m_{\mathrm{G}}^{h}}\left(\frac{\lambda^{a}}{2} \gamma_{\mu}\right)_{A C}\left(\frac{\lambda^{a}}{2} \gamma_{\mu}\right)_{D B} .
$$

A feature of the momentum independence of the CI is that the corresponding DS equation in Eq. (2) is non-renormalizable. In addition, the BS equation in the CI acquires ultraviolet divergencies and is also nonrenormalizable. This implies that mass-scale parameters introduced with the regularization of divergent integrals cannot be removed from the calculations and need to be fixed phenomenologically. Another feature, as previously mentioned, concerns the regularization of divergent integrals: they carry along the danger of symmetry violation, in particular the WGTI in Eq. (3) is not satisfied even when Poincaré-invariant regularization schemes are employed.

Let us consider the DS and the homogeneous pseudoscalar BS equations, Eqs. (2) and (4) respectively, with the CI approximation. In this case the solution of the gap equation becomes, $S_{f}^{-1}(k)=i \gamma \cdot k+M_{f}$, with the momentum independent quark-mass function,

$$
M_{f}=m_{f}+\frac{16}{3}\left(\frac{1}{m_{\mathrm{G}}^{f}}\right)^{2} \int_{q} \frac{M_{f}}{q^{2}+M_{f}^{2}} .
$$

It is remarkable that the non-running quark mass, $M_{f}$, is related to the commonplace constituent quark masses of quark and light-front models [56-60], yet it is dynamically generated. Such a momentum independent mass function is appropriate in the calculation of static observables, such as the meson mass spectrum, weak decay constants and charge radii, but leads to hard elastic and transition form factors that strongly depart from experimental data for $q^{2}>\Lambda_{\mathrm{QCD}}^{2}[26,32-40,42,61,62]$.

In this same CI framework, the pseudoscalar BS amplitude, for example, is independent of the relative quarkantiquark momentum. As a consequence, $G_{\mathrm{PS}}^{l h}=H_{\mathrm{PS}}^{l h}=$ 0, and Eq. (5) reduces to,

$$
\Gamma_{\mathrm{PS}}^{l h}(P)=\gamma_{5}\left[i E_{\mathrm{PS}}^{l h}(P)+\frac{1}{2 M_{l h}} \gamma \cdot P F_{\mathrm{PS}}^{l h}(P)\right],
$$

where $M_{l h}=M_{l} M_{h} /\left(M_{l}+M_{h}\right)$. Therefore, the BS equation can be written in the matrix form,

$$
\left[\begin{array}{c}
E_{\mathrm{PS}}^{l h}(P) \\
F_{\mathrm{PS}}^{l h}(P)
\end{array}\right]=\frac{1}{3 m_{\mathrm{G}}^{l} m_{\mathrm{G}}^{h}}\left[\begin{array}{ll}
\mathcal{K}_{\mathrm{PS}}^{E E} & \mathcal{K}_{\mathrm{PS}}^{E F} \\
\mathcal{K}_{\mathrm{PS}}^{F E} & \mathcal{K}_{\mathrm{PS}}^{F F}
\end{array}\right]\left[\begin{array}{c}
E_{\mathrm{PS}}^{l h}(P) \\
F_{\mathrm{PS}}^{l h}(P)
\end{array}\right],
$$


where the kernel's matrix elements are given by,

$$
\begin{aligned}
\mathcal{K}_{\mathrm{PS}}^{E E} & =-\int_{q} \operatorname{Tr}\left[\gamma_{5} \gamma_{\mu} S_{l}\left(q_{+}\right) \gamma_{5} S_{h}\left(q_{-}\right) \gamma_{\mu}\right] \\
\mathcal{K}_{\mathrm{PS}}^{E F} & =\frac{i}{2 M_{l h}} \int_{q} \operatorname{Tr}\left[\gamma_{5} \gamma_{\mu} S_{l}\left(q_{+}\right) \gamma_{5} \gamma \cdot P S_{h}\left(q_{-}\right) \gamma_{\mu}\right] \\
\mathcal{K}_{\mathrm{PS}}^{F E} & =\frac{2 i M_{l h}}{P^{2}} \int_{q} \operatorname{Tr}\left[\gamma_{5} \gamma \cdot P \gamma_{\mu} S_{l}\left(q_{+}\right) \gamma_{5} S_{h}\left(q_{-}\right) \gamma_{\mu}\right] \\
\mathcal{K}_{\mathrm{PS}}^{F F} & =\frac{1}{P^{2}} \int_{q} \operatorname{Tr}\left[\gamma_{5} \gamma \cdot P \gamma_{\mu} S_{l}\left(q_{+}\right) \gamma_{5} \gamma \cdot P S_{h}\left(q_{-}\right) \gamma_{\mu}\right]
\end{aligned}
$$

In Eqs. (11)-(14) the traces are over Dirac indices. All integrals in Eq. (8) and Eqs. (11)-(14) are ultraviolet divergent; the divergences are quadratic and logarithmic. The vast majority of applications within NJL models ignore the pseudo vector component $F_{\mathrm{PS}}^{l h}(P)$; in doing so leads to the random-phase-approximation (RPA) of the BS equation [22-25].

For vector mesons, the corresponding BS equation in the RL CI model is given by

$$
\Gamma_{\mathrm{V}}^{l h}(P)=\gamma_{\mu}^{\perp} E_{\mathrm{V}}^{l h}(P),
$$

where

$$
\gamma_{\mu}^{\perp}=\gamma_{\mu}-\frac{\gamma \cdot P}{P^{2}} P_{\mu}
$$

With only one Lorentz covariant the BS equation for the vector meson simplifies to

$$
1=\frac{1}{m_{G}^{l} m_{G}^{h}} K_{\mathrm{V}}^{E E}(P)
$$

with

$$
K_{\mathrm{V}}^{E E}(P)=-\frac{1}{3} \int_{q} \operatorname{Tr}\left[\gamma_{\nu}^{\perp} \gamma_{\mu} S_{l}\left(q_{+}\right) \gamma_{\nu}^{\perp} S_{h}\left(q_{-}\right) \gamma_{\mu}\right]
$$

\section{SYMMETRY-PRESERVING SUBTRACTION SCHEME}

The issue of symmetry violation can be exposed examining the momenta running in the quark propagators in the BS amplitudes in Eqs. (11)-(14); they are $q_{+}=q+\eta_{+} P$ and $q_{-}=q-\eta_{-} P$, where $\eta_{ \pm}$are arbitrary partition variables satisfying $\eta_{+}+\eta_{-}=1$. However, to maintain translational invariance, the results of the integrals can depend on the relative momentum $q_{+}-q_{-}$ only, or, equivalently, they must not depend on $\eta_{ \pm}$individually but solely on the combination $\eta_{+}+\eta_{-}=1$.
This dependence on the relative momentum is also crucial for preserving the WGTI in Eq. (3), as we discuss shortly ahead. Moreover, for very different values of $M_{h}$ and $M_{l}$, the independence of the results on $\eta_{+}$and $\eta_{-}$ is a serious issue in RL finite-range models [16] which becomes exacerbated in nonrenormalizable CI models.

Within NJL models, the customary way of handling integrals, such as in Eqs. (11)-(14), is as follows [22-25]: after evaluating the traces, a choice for $\eta_{+}$and $\eta_{-}$is made and Feynman parameters are used to combine in a single term the product $\left(q_{+}^{2}+M_{l}^{2}\right)\left(q_{-}^{2}+M_{h}^{2}\right)$ in the denominator. Thereafter a momentum shift is applied to eliminate the angle defined by the scalar product, $q \cdot P$, in the denominator and finally the integral over $q$ is performed. In shifting the momentum, changes in the integration limits are ignored. Invariably, results depend upon the choices made for $\eta_{ \pm}$; in particular, the value of the pion decay constant $f_{\pi}$, sensitive to the normalization of the pion BS equation, depends on the choices of the partition parameters. In some instances regularization-independent results can be obtained after using the gap equation to eliminate the quadratic divergences, like in the derivation of a Goldberg-Treiman relation at the quark level and the Gell-Mann-Oakes-Renner relationship - see e.g. the discussions around Eq. (4.27) in Ref. [23].

The subtraction scheme is based on the repeated use of the identity,

$$
\begin{aligned}
\frac{1}{q_{ \pm}^{2}+M_{l, h}^{2}} & =\left(\frac{1}{q_{ \pm}^{2}+M_{l, h}^{2}}-\frac{1}{q^{2}+M^{2}}\right)+\frac{1}{q^{2}+M^{2}} \\
& =\frac{1}{q^{2}+M^{2}}-\frac{\left(q_{ \pm}^{2}-q^{2}+M_{l, h}^{2}-M^{2}\right)}{\left(q^{2}+M^{2}\right)\left(q_{ \pm}^{2}+M_{l, h}^{2}\right)}
\end{aligned}
$$

where $M$ is an arbitrary subtraction mass-scale parameter. This mass scale plays a similar role to the $\mu$ scale in dimensional regularization and can be used to tune parameters of the model when applying it to explore physics at different scales. We do not trail this interesting possibility in this paper and simply keep $M$ arbitrary; we comment on this further ahead in this section.

Assuming a Poincaré-invariant regularization for the integrals in Eqs. (11)-(14), subtractions are performed in each of the propagators, $S_{l}\left(q_{+}\right)$and $S\left(q_{-}\right)$, the number of which is dictated by the requirement that a finite integral is obtained. Note that while the original denominator behaves as $1 / q^{2}$ in the limit $q \rightarrow \infty$, the last term in Eq. (19) tends to $1 / q^{4}$ for $q \rightarrow \infty$. We illustrate the procedure in detail for the $\mathcal{K}_{\mathrm{PS}}^{E E}(P)$ kernel. Evaluation of the trace in Eq. (11) leads to,

$$
\mathcal{K}_{\mathrm{PS}}^{E E}=16 \int_{q}^{\Lambda} \frac{q_{+} \cdot q_{-}+M_{l} M_{h}}{\left(q_{+}^{2}+M_{l}^{2}\right)\left(q_{-}^{2}+M_{h}^{2}\right)}=8 \int_{q}^{\Lambda}\left\{\frac{1}{q_{+}^{2}+M_{l}^{2}}+\frac{1}{q_{-}^{2}+M_{h}^{2}}-\frac{P^{2}+\left(\Delta M_{h l}\right)^{2}}{\left(q_{+}^{2}+M_{l}^{2}\right)\left(q_{+}^{2}+M_{h}^{2}\right)}\right\}
$$

where $\Delta M_{l h}=M_{l}-M_{h}$, and $\Lambda$ denotes the ultraviolet mass scale associated with the regularization. By using trice 
the identity of Eq. (19), the first two terms can be rewritten as

$$
\int_{q}^{\Lambda} \frac{1}{q_{ \pm}^{2}+M_{l, h}^{2}}=I_{\mathrm{quad}}\left(M_{l, h}^{2}\right)+\eta_{ \pm}^{2} P_{\mu} P_{\nu} A_{\mu \nu}\left(M^{2}\right),
$$

where $A_{\mu \nu}\left(M^{2}\right)$ is the integral defined by

$$
A_{\mu \nu}\left(M^{2}\right)=\int_{q}^{\Lambda} \frac{4 q_{\mu} q_{\nu}-\left(q^{2}+M^{2}\right) \delta_{\mu \nu}}{\left(q^{2}+M^{2}\right)^{3}},
$$

which is the Euclidean space counterpart of the integral $\Delta_{\mu \nu}\left(M^{2}\right)$ in Minkowski space of Refs. [43, 44, 50]. Using Eq. (21) and subtracting each of the denominators in the third term in Eq. (20), one can write $\mathcal{K}_{\mathrm{PS}}^{E E}$ can be written as a sum of three kinds of terms: (1) a finite integral independent of $\eta_{ \pm} ;(2)$ quadratic and logarithmically divergent integrals that are also independent of $\eta_{ \pm}$; and (3) a symmetry violating term proportional to $\eta_{+}^{2}$ and $\eta_{-}^{2}$, namely,

$$
\begin{aligned}
\mathcal{K}_{\mathrm{PS}}^{E E}= & 8\left\{-\left[P^{2}+\left(\Delta M_{h l}\right)^{2}\right]\left[I_{\log }\left(M^{2}\right)-Z_{0}\left(M_{l}^{2}, M_{h}^{2}, P^{2} ; M^{2}\right)\right]+I_{\text {quad }}\left(M_{l}^{2}\right)+I_{\text {quad }}\left(M_{h}^{2}\right)\right. \\
& \left.+\left(\eta_{+}^{2}+\eta_{-}^{2}\right) A_{\mu \nu}\left(M^{2}\right) P_{\mu} P_{\nu}\right\}
\end{aligned}
$$

where $Z_{0}\left(M_{l}^{2}, M_{h}^{2}, P^{2} ; M^{2}\right)$ is the finite integral

$$
Z_{0}\left(M_{l}^{2}, M_{h}^{2}, P^{2} ; M^{2}\right)=\int_{0}^{1} d z \int_{q}^{\Lambda}\left[\frac{1}{\left(q^{2}+M^{2}\right)^{2}}-\frac{1}{\left(q^{2}+H(z)\right)^{2}}\right]=\frac{1}{(4 \pi)^{2}} \int_{0}^{1} d z \ln \left[\frac{H(z)}{M^{2}}\right],
$$

where $H(z)$ is the function,

$$
H(z)=z(1-z) P^{2}-\left(M_{l}^{2}-M_{h}^{2}\right) z+M_{l}^{2} .
$$

Here $I_{\log }\left(M^{2}\right)$ and $I_{\text {quad }}\left(M^{2}\right)$ and are the logarithmically and quadratically divergent integrals,

$$
\begin{gathered}
I_{\log }\left(M^{2}\right)=\int_{q}^{\Lambda} \frac{1}{\left(q^{2}+M^{2}\right)^{2}}, \\
I_{\text {quad }}\left(M^{2}\right)=\int_{q}^{\Lambda} \frac{1}{q^{2}+M^{2}} .
\end{gathered}
$$

In the derivation of Eq. (23), we made use of the identity

$$
\begin{gathered}
I_{\text {quad }}\left(M_{l, h}^{2}\right)=I_{\text {quad }}\left(M^{2}\right)+\left(M_{l, h}^{2}-M^{2}\right) I_{\log }\left(M^{2}\right) \\
+\frac{1}{(4 \pi)^{2}}\left[M_{l, h}^{2}-M^{2}-M_{l, h}^{2} \ln \left(\frac{M_{l, h}^{2}}{M^{2}}\right)\right] .
\end{gathered}
$$

We note that to arrive at these results, no momentum shift was made in the divergent integrals; if one had shifted the momenta without change in the integration limits, one would have missed the term proportional to $A_{\mu \nu}\left(M^{2}\right)$. We also note that the divergences in each of the integrals in the first equality of Eq. (24) cancel and the final result is finite. Therefore, there is no need for a regulator in $Z_{0}$. This feature, that one can remove the regulator in finite integrals, plays a very important role when considering high temperatures and densities in the NJL model [46-49]. It will play an important role also in the phenomenology of mesons with heavy quarks, as we discuss in the next section.
We stress that Eq. (23) is an exact result and no approximations were made in the derivation from Eq. (20). Moreover, as already mentioned, no momentum shifts were made in obtaining the symmetry-violating and divergent integrals. This is important, as a momentum shift in a divergent integral is a delicate process and in many instances is the source of symmetry violation. Note that whatever choice made for $\eta_{ \pm}$unavoidably implies translation symmetry breaking, unless the regularization scheme leads to $A_{\mu \nu}\left(M^{2}\right)=0$. Momentum shifts were made only in the finite integral $Z_{0}\left(M_{l}^{2}, M_{h}^{2}, P^{2} ; M^{2}\right)$; it can be integrated without imposing an ultraviolet cutoff. The integral develops an imaginary part that reflects the possibility of meson decay into a quark-antiquark pair when $P^{2}<-\left(M_{l}+M_{h}\right)^{2}$; this is an unphysical feature that afflicts CI models. However, there is no difficulty in introducing an infrared cutoff [29] in this and other finite integrals to avoid unphysical quark-antiquark thresholds in BS amplitudes. This will be implemented in Sec. IV.

The expressions for the remaining kernels $\mathcal{K}_{\mathrm{PS}}^{E F}, \mathcal{K}_{\mathrm{PS}}^{F E}$, and $\mathcal{K}_{\mathrm{PS}}^{F F}$ contain the same terms as in Eq. (23) and an additional symmetry violation term - they are presented in Appendix A. We also note that one can use the gap equation, Eq. (8), to express the quadratically divergent integral, $I_{\text {quad }}$, in terms of the constituent-quark mass, $M_{f}$, as

$$
I_{\text {quad }}\left(M_{f}^{2}\right)=\frac{3}{16}\left(m_{\mathrm{G}}^{f}\right)^{2} \frac{\left(M_{f}-m_{f}\right)}{M_{f}} .
$$

We will make use of this equation further ahead.

Let us next examine how choices of $\eta_{ \pm}$lead to violation of the WGT identity in Eq. (3) in case of CI. In the chiral 
limit, $m^{h}=m^{l}=0$, combining Eq. (3) with Eq. (4), straightforward manipulations lead to two equations (for the light-light case):

$$
\begin{aligned}
& 0=M_{l}-\frac{8}{3}\left(\frac{1}{m_{\mathrm{G}}^{l}}\right)^{2} \int_{q}^{\Lambda}\left(\frac{M_{l}}{q_{+}^{2}+M_{l}^{2}}+\frac{M_{l}}{q_{-}^{2}+M_{l}^{2}}\right), \\
& 0=\int_{q}^{\Lambda}\left(\frac{q_{+} \cdot P}{q_{+}^{2}+M_{l}^{2}}-\frac{q_{-} \cdot P}{q_{-}^{2}+M_{l}^{2}}\right) .
\end{aligned}
$$

Using Eq. (21) in Eq. (30), one obtains,

$$
\begin{aligned}
0= & M_{l}-\frac{16}{3}\left(\frac{1}{m_{\mathrm{G}}^{l}}\right)^{2}\left[M_{l} I_{\text {quad }}\left(M_{l}^{2}\right)\right. \\
& \left.+M_{l}\left(\eta_{+}^{2}+\eta_{-}^{2}\right) A_{\mu \nu}\left(M^{2}\right) P_{\mu} P_{\nu}\right] .
\end{aligned}
$$

The last term, being proportional to $\eta_{+}^{2}$ and $\eta_{-}^{2}$ are symmetry-violating. The subtractions in Eq. (31) lead to new symmetry violating terms, in addition to terms proportional to $A_{\mu \nu}\left(M^{2}\right)$ :

$$
\begin{aligned}
\int_{q}^{\Lambda} \frac{q_{\mu}}{q_{ \pm}^{2}+M_{l}^{2}}= & \mp \eta_{ \pm} P_{\mu} I_{\text {quad }}\left(M_{l}^{2}\right) \mp \eta_{ \pm} P_{\alpha} B_{\alpha \mu}\left(M^{2}\right) \\
& \pm \eta_{ \pm}\left(\eta_{ \pm}^{2} P^{2}+M^{2}-M_{l}^{2}\right) P_{\alpha} A_{\alpha \mu}\left(M^{2}\right) \\
& \mp \frac{1}{2} \eta_{ \pm}^{3} P_{\alpha} A_{\alpha \beta}\left(M^{2}\right)\left(P_{\mu} P_{\beta}+P^{2} \delta_{\beta \mu}\right) \\
& \mp \frac{1}{3} \eta_{ \pm}^{3} P_{\alpha} P_{\beta} P_{\rho} C_{\alpha \beta \rho \mu}\left(M^{2}\right)
\end{aligned}
$$

where $B_{\mu \nu}\left(M^{2}\right)$ and $C_{\mu \nu \rho \sigma}\left(M^{2}\right)$ are new tensor structures,

$$
\begin{aligned}
B_{\mu \nu}\left(M^{2}\right) & =\int_{q}^{\Lambda} \frac{2 q_{\mu} q_{\nu}-\left(q^{2}+M^{2}\right) \delta_{\mu \nu}}{\left(q^{2}+M^{2}\right)^{2}}, \\
C_{\mu \nu \rho \sigma}\left(M^{2}\right) & =\int_{q}^{\Lambda} \frac{c_{\mu \nu \rho \sigma}\left(q, M^{2}\right)}{\left(q^{2}+M^{2}\right)^{4}}
\end{aligned}
$$

with

$$
\begin{aligned}
c_{\mu \nu \rho \sigma}\left(q^{2}, M^{2}\right) & =24 q_{\mu} q_{\nu} q_{\rho} q_{\sigma}-4\left(q^{2}+M^{2}\right) \\
& \times\left(\delta_{\mu \nu} q_{\rho} q_{\sigma}+\text { perm. } \nu \sigma \rho\right) .
\end{aligned}
$$

The $B_{\mu \nu}\left(M^{2}\right)$ and $C_{\mu \nu \rho \sigma}\left(M^{2}\right)$ integrals are the Euclidean space counterparts of the Minkowski space integrals $\nabla_{\mu \nu}\left(M^{2}\right)$ and $\square_{\mu \nu \rho \sigma}\left(M^{2}\right)$ of Refs. [43, 44, 50]. Using Eq. (33) in Eq. (31), we obtain,

$$
\begin{aligned}
0 & =\int_{q}^{\Lambda}\left(\frac{q_{+} \cdot P}{q_{+}^{2}+M_{l}^{2}}-\frac{q_{-} \cdot P}{q_{-}^{2}+M_{l}^{2}}\right) \\
& =\text { terms proportional to } \eta_{ \pm}\left(A_{\mu \nu}, B_{\mu \nu}, C_{\mu \nu \rho \sigma}\right) .
\end{aligned}
$$

We observe that for arbitrary momentum routing in the loop integrals, the subtraction scheme allows to systematically identify symmetry violating terms; they are proportional to the integrals $A_{\mu \nu}, B_{\mu \nu}$ and $C_{\mu \nu \rho \sigma}$ in Eqs. (22),
(34) and (35). A consistent regularization scheme must make the integrals vanish automatically. Otherwise, the vanishing of the integrals must be imposed; in doing so, the regularization scheme becomes a central part of the model. Dimensional regularization and Pauli-Villars regularization are examples of schemes that lead to $A_{\mu \nu}=0$, $B_{\mu \nu}=0$, and $C_{\mu \nu \rho \sigma}=0$. Removing the symmetryviolating terms, Eq. (32) becomes nothing else than the gap equation of Eq. (8).

Let us make contact with the GBCR scheme [26]. For a proper comparison, we need to take $\eta_{+}=1$ and $\eta_{-}=$ 0 , the choice made in that reference. For this choice, Eq. (31) becomes

$$
\int_{q}^{\Lambda}\left(\frac{q_{+} \cdot P}{q_{+}^{2}+M_{l}^{2}}-\frac{q \cdot P}{q^{2}+M_{l}^{2}}\right)=0,
$$

which is Eq. (15) of Ref. [26]. Using Eq. (33) for this integral, one obtains:

$$
\begin{aligned}
0= & \int_{q}^{\Lambda}\left(\frac{q_{+} \cdot P}{q_{+}^{2}+M^{2}}-\frac{q \cdot P}{q^{2}+M^{2}}\right) \\
= & P_{\mu}\left[B_{\mu \nu}\left(M^{2}\right)+\frac{1}{3} C_{\mu \nu \rho \sigma}\left(M^{2}\right) P_{\rho} P_{\sigma}\right. \\
& \left.+\frac{1}{3} P_{\mu} A_{\rho \nu}\left(M^{2}\right) P_{\rho}-\frac{4}{3} P^{2} A_{\mu \nu}\left(M^{2}\right)\right] P_{\nu},
\end{aligned}
$$

that is, the $M_{l}$ dependence cancels and the remaining divergent terms depend on $M$ only:

$$
\begin{aligned}
B_{\mu \nu}( & \left.M^{2}\right)+\frac{1}{3} C_{\mu \nu \rho \sigma}\left(M^{2}\right) P_{\rho} P_{\sigma} \\
+ & \frac{1}{3} P_{\mu} A_{\rho \nu}\left(M^{2}\right) P_{\rho}-\frac{4}{3} P^{2} A_{\mu \nu}\left(M^{2}\right)=0 .
\end{aligned}
$$

In the chiral limit, this is equal to

$$
B_{\mu \nu}\left(M^{2}\right)=0=\delta_{\mu \nu} \int_{\Lambda} \frac{d^{4} q}{(2 \pi)^{4}} \frac{\frac{1}{2} q^{2}+M^{2}}{\left(q^{2}+M^{2}\right)^{2}},
$$

which is Eq. (17) of Ref. [26]. This result makes it clear that our scheme, besides being in agreement with Ref. [26] for the particular choice of $\eta_{ \pm}$, it is also more general as is valid for arbitrary values of $\eta_{ \pm}$. This is important when $M_{h} \gg M_{l}$, as results are very sensitive to the momentum partitioning between the heavy and light quarks in a bound state [16]; for $u$ and $s$ quarks, it has been shown [63] that results for $\pi$ and $K$ observables are not very sensitive to this momentum partitioning.

Here, we take the opportunity to comment on the role played by the subtraction mass $M$. As mentioned previously, it shares similarities with the mass scale $\mu$ that appears in dimensional regularization. Indeed, suppose one uses dimensional regularization in the model. Besides removing automatically all potential symmetryviolating terms, dimensional regularization introduces an arbitrary mass scale $\mu$ that comes with the replacement $D=4 \rightarrow D=4-2 \epsilon$ in the integrals. On the other 
hand, in the subtraction scheme one introduces the arbitrary mass $M$ and prescribe that symmetry-violating terms vanish. In addition, one also obtains divergent integrals depending on $M$, like $I_{\log }\left(M^{2}\right)$ and $I_{\text {quad }}\left(M^{2}\right)$ of Eqs. (26) and (27), that need a regularization that cannot be removed because the model is nonrenormalizable. Applying dimensional regularization in those integrals, one introduces two arbitrary dimensionful parameters, $M$ and $\mu$ :

$$
\begin{aligned}
I_{\log }\left(M^{2}\right) & =(\mu)^{2 \epsilon} \int \frac{d^{D} q}{(2 \pi)^{D}} \frac{1}{\left(q^{2}+M^{2}\right)^{2}} \\
& =\frac{1}{(4 \pi)^{2}}\left[\frac{1}{\epsilon}-\ln \left(\frac{M^{2}}{\bar{\mu}^{2}}\right)\right],
\end{aligned}
$$

where $\bar{\mu}^{2}=4 \pi e^{-\gamma} \mu^{2}$. Choosing $\bar{\mu}=M$, the integral is scale independent; likewise for $I_{\text {quad }}\left(M^{2}\right)$, it depends only on $M$. Alternatively, there is no need for evaluating these integrals with an explicit regulator, since $M$ can be set by fitting the parameters of the model to physical quantities [43-45]. Through relations like that in Eq. (28), one obtains the divergent integrals at any other scale in terms of the physical quantities fitted at the scale $M$. As said, we do not trail this interesting possibility in this paper and simply keep $M$ arbitrary and use a second scale that is an ultraviolet cutoff. As we shall discuss in the next section, our results are essentially independent of $M$; small differences in the results caused by using different values can be absorbed by refitting parameters.

The masses of the mesons are obtained from Eq. (10); the equation defines an eigenvalue problem with solutions for the masses $P^{2}=-m_{\mathrm{PS}}^{2}$, namely:

$$
\lambda\left(P^{2}\right) \Gamma_{\mathrm{PS}}\left(P^{2}\right)=\mathcal{K}\left(P^{2}\right) \Gamma_{\mathrm{PS}}\left(P^{2}\right),
$$

where $\mathcal{K}\left(P^{2}\right)$ is the $2 \times 2$ matrix defined in Eq. (10), and $\lambda\left(-m_{\mathrm{PS}}^{2}\right)=1$. The derivation of the subtracted kernels is detailed in Appendix A.

The weak decay constant of the PS meson, $f_{\mathrm{PS}}$, can be extracted from:

$$
\begin{aligned}
P_{\mu} f_{\mathrm{PS}} & =\left\langle 0\left|\bar{\psi}_{l}(0) \gamma_{\mu} \gamma_{5} \psi_{h}(0)\right| \phi(P)\right\rangle \\
& =N_{c} \int_{q} \operatorname{Tr}\left[\gamma_{5} \gamma_{\mu} S_{l}\left(q_{+}\right) \Gamma_{\mathrm{PS}}^{l h}(P) S_{h}\left(q_{-}\right)\right],
\end{aligned}
$$

where the trace is over Dirac indices. Inserting Eq. (9) into Eq. (44) and evaluating the trace leads to,

$$
f_{\mathrm{PS}}=\frac{N_{c}}{4 M_{l h}}\left[E_{\mathrm{PS}}(P) \mathcal{K}_{\mathrm{PS}}^{F E}+F_{\mathrm{PS}}(P) \mathcal{K}_{\mathrm{PS}}^{F F}\right]_{P^{2}=-m_{\mathrm{PS}}^{2}},
$$

where, we recall, $M_{l h}=M_{l} M_{h} /\left(M_{l}+M_{h}\right)$. The eigenvalue equation in Eq. (43) does not fix the amplitudes $E_{\mathrm{PS}}(P)$ and $F_{\mathrm{PS}}(P)$ separately; for that, one needs a normalization condition, which we take as,

$$
\begin{aligned}
2 P_{\mu}= & N_{c} \int \frac{d^{4} q}{(2 \pi)^{4}} \operatorname{Tr}\left[\Gamma_{\mathrm{PS}}^{h l}(-P) \frac{\partial S_{l}\left(q_{+}\right)}{\partial P_{\mu}} \Gamma_{\mathrm{PS}}^{h l}(P) S_{h}\left(q_{-}\right)\right. \\
& \left.+\Gamma_{\mathrm{PS}}^{h l}(-P) S_{l}\left(q_{+}\right) \Gamma_{\mathrm{PS}}^{h l}(P) \frac{\partial S_{h}\left(q_{-}\right)}{\partial P_{\mu}}\right]
\end{aligned}
$$

evaluated at $P^{2}=-m_{\mathrm{PS}}^{2}$. We note that with this convention, the experimental value of the pion decay constant is $f_{\pi} \simeq 130 \mathrm{MeV}$ [64].

To close this section, we comment on the regularization procedure. On computing the masses and the decay constants of the pseudoscalar mesons, the first step is to obtain the constituent quark masses, $M_{f}$, from the gap equation, Eq. (8), and then solve the eigenvalue problem, Eq. (43). Both equations contain the ultraviolet divergent integrals $I_{\text {quad }}$ and $I_{\log }$ that need regularization and, in addition, Eq. (43) contains also the finite integral $Z_{0}$-function (and the $Z_{1}$ that appears in the other kernels). As briefly discussed in Sec. III, $Z_{0}$ develops thresholds as it can acquire an imaginary part when $P^{2}<-\left(M_{l}+M_{h}\right)^{2}$. We avoid the possibility of unphysical thresholds in hadron decay into quarks by eliminating this branch cut with an infrared cutoff. Both ultraviolet and infrared cutoffs can be introduced using a proper-time regularization [29] as follows. All integrals, finite and divergent, contain integrands of the form $1 /\left(q^{2}+a^{2}\right)^{n}$, where $n=1,2$ and $a^{2}$ is independent of $q$. The imaginary part in $Z_{0}$ happens when $q^{2} \rightarrow-a^{2}$; to avoid this to happen, one then rewrites the integrands as

$$
\begin{aligned}
\frac{1}{\left(q^{2}+a^{2}\right)^{n}} & =\frac{1}{(n-1) !} \int_{0}^{\infty} d \tau \tau^{n-1} e^{-\tau\left(q^{2}+a^{2}\right)} \\
& \rightarrow \frac{1}{(n-1) !} \int_{\tau_{\mathrm{uv}}}^{\tau_{\mathrm{ir}}} d \tau \tau^{n-1} e^{-\tau\left(q^{2}+a^{2}\right)},
\end{aligned}
$$

with $\tau_{\mathrm{uv}}=1 / \Lambda_{\mathrm{uv}}^{2}$ and $\tau_{\mathrm{ir}}=1 / \Lambda_{\mathrm{uv}}^{2}$, where $\Lambda_{\mathrm{uv}}$ and $\Lambda_{\mathrm{ir}}$ are the ultraviolet and infrared cutoffs respectively. For the $Z_{0}$ integral, $\tau_{\mathrm{uv}}$ is not needed, as the integral is finite; therefore

$$
\frac{1}{\left(q^{2}+a^{2}\right)^{2}} \rightarrow \frac{1-\left[1+\left(q^{2}+a^{2}\right) \tau_{\mathrm{ir}}\right] e^{-\tau_{\mathrm{ir}}\left(q^{2}+a^{2}\right)}}{\left(q^{2}+a^{2}\right)^{2}},
$$

which is finite when $q^{2} \rightarrow-a^{2}$, as can be verified very easily. The final result for $Z_{0}$ can be written as

$$
\begin{aligned}
Z_{0}\left(M_{l}^{2}, M_{h}^{2}, P^{2} ; M^{2}\right)= & \frac{1}{(4 \pi)^{2}} \int_{0}^{1} d z \int_{0}^{\tau_{\mathrm{ir}}^{2}} \frac{d \tau}{\tau} e^{-\tau M^{2}} \\
& \times\left(1-e^{-\tau\left[H(z)-M^{2}\right]}\right),
\end{aligned}
$$

which is clearly finite when $\tau \rightarrow 0$. Unphysical thresholds in the function $Z_{1}$ are eliminated using its relation to $Z_{0}$, given in Eq. (B2).

Although $\tau_{\text {ir }}$ is not needed for $I_{\text {quad }}$ and $I_{\log }$, we keep it in those integrals to compare results with Ref. [26]. Therefore

$$
I_{\text {quad }}\left(M_{f}^{2}\right)=\frac{1}{(4 \pi)^{2}} \int_{\tau_{\mathrm{uv}}^{2}}^{\tau_{\mathrm{ir}}^{2}} \frac{d \tau}{\tau^{2}} e^{-\tau M_{f}^{2}} .
$$

The logarithmically divergent integral $I_{\log }\left(M_{f}^{2}\right)$ can be derived from $I_{\text {quad }}\left(M_{f}^{2}\right)$ by a simple differentiation:

$$
I_{\log }\left(M_{f}^{2}\right)=-\frac{\partial I_{\text {quad }}\left(M_{f}^{2}\right)}{\partial M_{f}^{2}} .
$$




\section{NUMERICAL RESULTS}

We start considering the $f=\{u, d, s\}$ quark flavors and compare results from the CI subtraction and GBCR schemes [26]. More specifically, we compare results with Ref. [36] that extended the GBCR CI to the strange flavor sector. We recall the differences that mark both approaches: in the subtraction scheme the results are independent of $\eta_{ \pm}$, while those in Refs. $[26,36]$ are for $\eta_{+}=1$ and $\eta_{-}=0$. The finite integrals $Z_{0}$ and $Z_{1}$ in the BS amplitudes that appear in the subtraction scheme can be integrated without imposing ultraviolet or infrared cutoffs, though we removed the thresholds of these functions by means of infrared cutoff as discussed in the previous section. As shown in Sec. III, for $\eta_{+}=1$ and $\eta_{-}=0$ and neglecting symmetry violating terms and using the same ultraviolet and infrared cutoffs, both approaches lead to identical results.

The free parameters are the quark masses $m_{u}, m_{d}$ and $m_{s}$, the coupling strength $\alpha_{\mathrm{IR}}$, the gluon-mass scale $m_{g}$, and the cutoffs $\Lambda_{\text {ir }}$ and $\Lambda_{\text {uv }}$. For a proper comparison, we use the parameter set of Ref. [36]: $m_{u}=m_{d}=m=$ $7 \mathrm{MeV}, m_{s}=170 \mathrm{MeV}, \alpha_{\mathrm{IR}}=0.93 \pi, m_{g}=800 \mathrm{MeV}$, $\Lambda_{\mathrm{ir}}=240 \mathrm{MeV}$, and $\Lambda_{\mathrm{uv}}=905 \mathrm{MeV}$. The ultraviolet cutoff is used in the divergent integrals $I_{\log }$ and $I_{\text {quad }}$ only. These parameters give for the constituent masses, solutions of the gap equation in Eq. (8): $M_{u}=M_{d}=$ $367 \mathrm{MeV}$ and $M_{s}=523 \mathrm{MeV}$.

In Tab. I we present the BS amplitudes $(E, F)_{\mathrm{PS}}$ and masses and decay constants $(m, f)_{\mathrm{PS}}$ of $\pi$ and $K$ from both approaches. Here, we use the natural value for the subtraction mass $M$ in the calculation of for both $\pi$ and $K$ properties, namely the light constituent quark mass, $M=M_{u}$; choosing $M=M_{s}$, or any other value between $M_{u}$ and $M_{s}$, does not change the results for the properties of $\pi$ and $K$, as expected. Table I informs that the results in both approaches compare very well, and are in good agreement with experimental data. The particular choice of momentum partitioning and use or not of an ultraviolet cutoff in finite integrals does not affect the results in this case. While the first conclusion is consistent with the findings in Ref. [63] in a finite-range RL model, the second means that the momentum integrands that give rise to the finite functions $Z_{0}$ and $Z_{1}$ have integrands well concentrated within the momentum range extending from $\Lambda_{\mathrm{ir}}$ to $\Lambda_{\mathrm{uv}}$.

Next, we include the charm quark and calculate properties of heavy-light mesons. The issue on the necessity for a flavor dependence of the effective coupling is exposed: using the same parameters as in the light quark sector and adjusting the charm quark mass to fit the mass of the $D$-meson, the decay constant $f_{D}$ turns out smaller than $f_{K}$. The same feature is also present in Ref. [53] within the NJL model. Like in the extension of the NJL model made in Refs. $[46,55]$ to high temperatures and densities, and also in the extension of the GBCR CI model in Refs. [41, 42] to charmonium, one can readjust the effective coupling $m_{\mathrm{G}}^{h}$ and the ultravi-
TABLE I. BS amplitudes $(E, F)_{\mathrm{PS}}$ and masses and decay constants $(m, f)_{\mathrm{PS}}$ of $\pi$ and $K$ from the CI subtraction scheme and CI GBCR of Ref. [36]. The amplitudes are dimensionless and masses and decay constants are listed in $\mathrm{MeV}$.

\begin{tabular}{lcccc}
\hline \hline & $(E, F)_{\pi}$ & $(E, F)_{K}$ & $(m, f)_{\pi}$ & $(m, f)_{K}$ \\
\cline { 2 - 5 } Data [64] & - & - & $(139,131)$ & $(494,156)$ \\
CI-subtr & $(5.10,0.67)$ & $(5.64,0.85)$ & $(139,144)$ & $(502,153)$ \\
GBCR [36] & $(5.09,0.68)$ & $(5.40,0.83)$ & $(140,141)$ & $(500,156)$ \\
\hline \hline
\end{tabular}

olet cutoff $\Lambda_{\mathrm{uv}}$ in our model. In the light quark sector, we used $m_{g}=800 \mathrm{MeV}$ and $\alpha_{\mathrm{IR}}=0.93 \pi$, which imply $m_{\mathrm{G}}^{l}=132 \mathrm{MeV}$. We set $m_{c}=1454 \mathrm{MeV}$, and reset $\Lambda_{\mathrm{uv}}=1290 \mathrm{MeV}$ and $m_{\mathrm{G}}^{h}=3.5 m_{\mathrm{G}}^{l}=462 \mathrm{MeV}$ - see Eq. 7. We stress that the new value of $\Lambda_{\mathrm{uv}}$ is used in the gap and BS equation involving the charm quark only. Regarding the subtraction mass $M$, we use for it the light quark mass; more on this at the end of this section. From the gap equation, we obtain $M_{s}=529 \mathrm{MeV}$ and $M_{c}=1490 \mathrm{MeV}$, which shows that there is very little dressing of the charm quark.

TABLE II. Masses and decay constants of the pseudoscalar mesons (in $\mathrm{MeV}$ ). Parameters of the CI subtraction model are given in the main text. NST1, NST2, REBM1, REBM2, HGKL1 and HGKL2 are results from representative studies employing finite-range interactions treated within the RL framework of the DS and BS equations in QCD.

\begin{tabular}{lrrrc}
\hline \hline & \multicolumn{4}{c}{$(m, f)_{\mathrm{PS}}$} \\
\cline { 2 - 5 } & $\pi$ & \multicolumn{4}{c}{$\mathrm{K}$} & $D_{s}$ \\
\hline Data [64] & $(139,131)$ & $(494,156)$ & $(1864,212)$ & $(1968,249)$ \\
CI-subtr & $(139,143)$ & $(494,153)$ & $(1869,207)$ & $(1977,240)$ \\
GBCR [34] & $(140,141)$ & $(500,156)$ & - & - \\
NJL [53] & $(135,131)$ & $(498,135)$ & $(1869,113)$ & - \\
NST1 [9] & $(138,131)$ & $(497,155)$ & $(1850,222)$ & $(1970,197)$ \\
NST2 [9] & - & - & $(1880,260)$ & $(1900,275)$ \\
REBM1 [14] & $(138,139)$ & $(493,164)$ & $(2115,204)$ & $(2130,249)$ \\
REBM2 [14] & $(153,189)$ & $(541,214)$ & $(2255,281)$ & $(2284,320)$ \\
HGKL1 [17] & $(137,133)$ & $(492,155)$ & $(1868,323)$ & $(1872,269)$ \\
HGKL2 [17] & $(137,128)$ & $(489,150)$ & $(1869,960)$ & $(1802,295)$ \\
\hline \hline
\end{tabular}

Table. II presents our results for the masses and decays constants of pseudoscalar mesons. We have also readjusted the strange quark mass to $m_{s}=165 \mathrm{MeV}$ to obtain a perfect fit to $m_{K}$. For orientation and comparison, we have listed in the table the results from the GBCR CI and from several representative studies employing finite-range interactions treated within the RL framework of the DS and BS equations in QCD. The latter are identified in the table by NST1 and NST2 from Ref. [9], REBM1 and REBM2 from Ref. [14] and 
HGKL1 and HGKL2 from Ref. [17]. The first observation one can draw from Tab. II is that, once the parameters of CI subtraction model are fixed to obtain an almost perfect agreement with experiment for the $m_{\text {PS }}$ masses, the model predicts for the decay constants, $f_{\pi}<f_{K}<f_{D}<f_{D_{s}}$, a pattern that is corroborated by realistic DS-BS and lattice-QCD calculations. Moreover, the individual values of $f_{\mathrm{PS}}$ are in very good agreement with the experimental and lattice data collected by the PDG [64], the discrepancies being at the level of a few percent. It is also remarkable that the CI subtraction model gives a much better description for $(m, f)_{\mathrm{PS}}$ than finite-range models within the RL framework, in which the discrepancies with the PDG values for the decay constants can reach up to $40 \%$ in some cases.

Regarding the very small value of $f_{D}$ in the NJL model, one may reasonably object that it is due to the inadequate use of parameters adjusted to the light mesons. Although it is true that one can remedy this situation adjusting the coupling and cutoff in the NJL model, one still faces the problem that the results become unacceptably dependent on the choices for $\eta_{ \pm}$. This means that for any new choice of $\eta_{ \pm}$, new values for the parameters of the model are required to fit observables; the situation is even more dramatic, in that no solutions for the BS equation can be found for some choices of $\eta_{ \pm}$. The subtraction scheme solves this problem.

We have also calculated masses and decay constants of vector mesons with the same parameter set used for the pseudoscalar mesons; the results are listed in Tab. III. There is fairly good experimental information on their masses but not on their decay constants. The values quoted in Tab. III for the masses are taken from PDG [64] and those for the decay constantes of $\rho$ and $K^{*}$ are extracted indirectly [65] from $\tau$ decays, whereas those for $D^{*}$ and $D_{s}^{*}$ are from a recent lattice calculation [66]. The CI predictions for the masses, $m_{\mathrm{V}}$, agree very well with experiment but less well for $f_{\mathrm{V}}$, as there is a wrong ordering between $f_{\rho}$ and $f_{K^{*}}$ and between $f_{D^{*}}$ and $f_{D_{s}^{*}}$. On the other hand, inspection of Tab. III reveals that finite-range RL models also have difficulties in the vector sector; for instance, $f_{D_{s}^{*}}<f_{K^{*}}$ in all of those models. In this connection, also calculated charmonium properties: in the same vein of Ref. [41], by a further increase of $m_{G}^{h}$ and $\Lambda_{\mathrm{uv}}$, the CI subtraction model predicts similar results to those of the GBCR model, including the wrong ordering between the vector and pseudoscalar decay constants, $f_{J / \Psi}<f_{\eta_{c}}$. This is an additional indication that the vector channels pose a challenge for CI models, possibly reflecting in this context the limitations of the RL framework exposed in finite-range models $[6-8,12]$.

\section{SUMMARY AND CONCLUSION}

We examined the CI model introduced in Ref. [26] within the perspective of a regularization scheme that allows to separate symmetry-violating parts in BS am-
TABLE III. Masses and decay constants of vector mesons (in $\mathrm{MeV}$ ). Parameters of the CI subtraction model are the same as in Tab. II. The entries for Data are explained in the text.

\begin{tabular}{lcccc}
\hline \hline & \multicolumn{4}{c}{$(m, f)_{V}$} \\
\cline { 2 - 5 } & $\rho$ & $K^{*}$ & $D^{*}$ & $D_{s}^{*}$ \\
\cline { 2 - 5 } & & & & \\
Data & $(775,212)$ & $(892,225)$ & $(2010,278)$ & $(2112,322)$ \\
CI-subt & $(776,205)$ & $(881,195)$ & $(2011,281)$ & $(2098,276)$ \\
GBCR [36] & $(930,-)$ & $(1030,-)$ & - & - \\
GBCR [33] & $(928,182)$ & $(-,-)$ & - & - \\
NST1 [9] & $(742,207)$ & $(936,241)$ & $(2040,160)$ & $(2170,180)$ \\
HGKL1 [17] & $(758,219)$ & $(946,247)$ & - & $(2175,178)$ \\
HGKL2 [17] & $(725,203)$ & $(919,237)$ & - & - \\
\hline \hline
\end{tabular}

plitudes in a choice independent of the momentum partition between the quark and antiquark in the bound state. In doing so, spacetime-translation symmetry and the WGT identities reflecting global symmetries of the model are preserved by the regularization. Symmetryoffending parts of the amplitudes, the integrals $A_{\mu \nu}, B_{\mu \nu}$, $C_{\mu \nu}$ and $D_{\mu \nu}$, can be neatly separated. In general, a cutoff regularization scheme leads to nonzero values for the symmetry-violating integrals, while dimensional regularization leads to the vanishing of the symmetry-offending integrals. In a nonrenormalizable model, like the contactinteraction model discussed here, the vanishing of $A_{\mu \nu}$, $B_{\mu \nu}$ and $C_{\mu \nu}$ must be imposed in an ad hoc manner and te imposition becomes an integral part of the model.

In order to check the subtraction scheme we have studied properties of the heavy-light $D, D_{s}, D^{*}$ and $D_{s}$ mesons, for which symmetry offending terms in a CI model have catastrophic consequences, as well as of light mesons $\pi, K, \rho$ and $K^{*}$. We have shown that using the subtraction scheme we can obtain values for the masses of all of these mesons that agree well with experiment. In addition, we can obtain the correct trend of the weak decay constants of the pseudoscalar mesons, $f_{\pi}<f_{K}<f_{D}<f_{D_{s}}$, and their individual values are also in good agreement with experiment and lattice QCD simulations. The values of the decay constants of the $\rho$ and $D^{*}$ agree very well with experiment and lattice QCD simulations, respectively; but the model predicts the wrong orderings $f_{\rho}<f_{K}$ and $f_{D^{*}}<f_{D_{s}^{*}}$. These difficulties in the vector sector might not be related to intrinsic deficiencies of a contact interaction, as similar deficiencies have been observed in RL calculations using the DS-BS equations employing sophisticated finiterange interaction kernels.

We reiterate that the CI subtraction scheme developed here is not meant to be a substitute for a full-fledged, QCD-based DS-BS framework. Rather, its aspiration is to be a simpler tool that preserves spacetime symmetries and global symmetries of QCD. We strongly believe that 
a simpler tool that respects such basic symmetries, and at the same time, is able to give comparable results of more sophisticated and intricate approaches, acquires the necessary credentials to be used to explore and provide useful insight into complex problems of current interest, like multihadron molecules and hadronic matter at finite temperature and density.

As a next application of the subtraction scheme we mention the study of strong couplings of charmed hadrons with light hadrons, in particular the couplings of $D$-mesons to nucleons. Such couplings are relevant in studies of the $D$-nucleon interaction at low energies [6770], $D$-mesic nuclei [71-73], $J / \psi$ binding to nuclei [7476], among others. There is no experimental information about the $D$-nucleon interaction, most of the knowledge on this interaction comes from calculations using hadronic Lagrangians motivated by SU(4) extensions of light-flavor chiral Lagrangians. Since the symmetry is badly broken by the widely different values of the quark masses, it is important to check the validity of SU(4) symmetry in the couplings not only in vacuum [77-81], but at finite temperature and density as well. Another future application is to study charm meson diffusion in mesonic matter. Here it is necessary to compute for example the temperature dependence of masses of $D^{+}\left(D^{-}\right)$mesons and also the light mesons $\pi, K$ and $\eta$ [82]. With these inputs, one is able to compute the drag and diffusion of $D^{+}$meson in hadronic medium.

\section{ACKNOWLEDGMENTS}

This work of was supported in part by Conselho Nacional de Desenvolvimento Científico e Tecnológico - CNPq, Grants nos. 140041/2014-1 (F.E.S.), 458371/2014-9 (B.E.-B), 305894/2009-9 (G.K.), Fundação de Amparo à Pesquisa do Estado de São Paulo - FAPESP, Grant No. 2013/01907-0 (G.K), 2016/031547 (B.E.-B.) and by a doctoral scholarships by Coordenação de Aperfeiçoamento de Pessoal de Nível Superior - CAPES (F.E.S.).

\section{Appendix A: Bethe-Salpeter kernels}

Here we collect the expressions of the Bethe-Salpeter kernels for pseudoscalar and vector and vector channels.

\section{Pseudoscalar kernels}

After taking Dirac traces and performing the necessary number of subtractions in Eqs. (11)-(14), we arrive at the expressions.

$$
\begin{aligned}
\mathcal{K}_{\mathrm{PS}}^{E F}= & \frac{P^{2}}{2 M_{l h}^{2}} \mathcal{K}_{\mathrm{PS}}^{F E}=\frac{8 P^{2}}{M_{l h}}\left\{\frac{M_{h}+M_{l}}{2} I_{\log }\left(M^{2}\right)+\left(M_{l}-M_{h}\right) Z_{1}\left(M_{l}^{2}, M_{h}^{2}, P^{2} ; M^{2}\right)-M_{l} Z_{0}\left(M_{l}^{2}, M_{h}^{2}, P^{2} ; M^{2}\right)\right\} \\
& +\frac{4 A_{\mu \nu}\left(M^{2}\right)}{M_{l h}}\left(M_{l}-M_{h}\right) P_{\mu} P_{\nu}\left(\eta_{+}-\eta_{-}\right), \\
\mathcal{K}_{\mathrm{PS}}^{F F}= & 8 \frac{\left(M_{l}^{2}-M_{h}^{2}\right)}{2 P^{2}}\left[I_{\text {quad }}\left(M_{h}^{2}\right)-I_{\text {quad }}\left(M_{l}^{2}\right)\right]-\frac{8\left(M_{l}+M_{h}\right)^{2}\left[P^{2}+\left(M_{l}-M_{h}\right)^{2}\right]}{2 P^{2}} \\
& \times\left[I_{\log }\left(M^{2}\right)-Z_{0}\left(M_{l}^{2}, M_{h}^{2}, P^{2} ; M^{2}\right)\right]+\frac{8}{P^{2}} P_{\mu} P_{\nu} D_{\mu \nu}\left(M^{2}\right),
\end{aligned}
$$

where $D_{\mu \nu}\left(M^{2}\right)$ is given by

$$
\begin{aligned}
D_{\mu \nu}\left(M^{2}\right) & =B_{\mu \nu}\left(M^{2}\right)-\frac{\left(\eta_{+}-\eta_{-}\right)^{2} P_{\mu} P_{\alpha}}{2} A_{\alpha \nu}\left(M^{2}\right)-\frac{\left(\eta_{+}-\eta_{-}\right)^{2} P_{\nu} P_{\beta}}{2} A_{\beta \mu}\left(M^{2}\right) \\
& -\frac{\left[P^{2}\left(\eta_{+}^{2}-\eta_{-}^{2}\right)+M_{h}^{2}-M_{l}^{2}\right]}{2} A_{\mu \nu}\left(M^{2}\right)+\frac{\left(\eta_{+}^{2}+\eta_{-}^{2}-\eta_{+} \eta_{-}\right) P_{\alpha} P_{\beta}}{3}\left[C_{\alpha \beta \mu \nu}\left(M^{2}\right)\right. \\
& \left.+\delta_{\alpha \beta} A_{\mu \nu}\left(M_{h}^{2}\right)+\delta_{\alpha \mu} A_{\beta \nu}\left(M^{2}\right)+\delta_{\alpha \nu} A_{\mu \beta}\left(M^{2}\right)\right]-2 P_{\alpha} P_{\beta}\left[\eta_{+}^{2} A_{\alpha \beta}\left(M^{2}\right)+\eta_{-}^{2} A_{\alpha \beta}\left(M^{2}\right)\right] .
\end{aligned}
$$

For the normalization of the BS amplitudes $E_{\mathrm{PS}}^{l h}$ and $F_{\mathrm{PS}}^{l h}$, stated by Eq. (46) we get

$$
1=-\left.\frac{3}{2}\left\{\left(E_{\mathrm{PS}}^{l h}\right)^{2} \frac{\partial \mathcal{K}_{\mathrm{PS}}^{E E}}{\partial P^{2}}+2 E_{\mathrm{PS}}^{l h} F_{\mathrm{PS}}^{l h}\left[-\frac{1}{2 P^{2}} \mathcal{K}_{\mathrm{PS}}^{E F}+\frac{\partial \mathcal{K}_{\mathrm{PS}}^{E F}}{\partial P^{2}}\right]+\frac{\left(F_{\mathrm{PS}}^{l h}\right)^{2} P^{2}}{2 M_{l h}^{2}} \frac{\partial \mathcal{K}_{\mathrm{PS}}^{F F}}{\partial P^{2}}\right\}\right|_{P^{2}=-m_{\mathrm{PS}}^{2}},
$$


with the derivatives of the kernels given by the following expressions:

$$
\begin{aligned}
\frac{\partial \mathcal{K}_{\mathrm{PS}}^{E E}}{\partial P^{2}}= & -8\left[I_{\log }\left(M^{2}\right)-Z_{0}\left(M_{l}^{2}, M_{h}^{2}, P^{2} ; M^{2}\right)\right]-8\left[P^{2}+\left(M_{l}-M_{h}\right)^{2}\right] Y_{1}\left(M_{l}^{2}, M_{h}^{2}, P^{2} ; M^{2}\right) \\
\frac{\partial \mathcal{K}_{\mathrm{PS}}^{E F}}{\partial P^{2}}= & \frac{\mathcal{K}_{\mathrm{PS}}^{E F}}{P^{2}}+\frac{8 P^{2}}{M_{l h}}\left[\left(M_{l}-M_{h}\right) Y_{2}\left(M_{l}^{2}, M_{h}^{2}, P^{2} ; M^{2}\right)-M_{l} Y_{1}\left(M_{l}^{2}, M_{h}^{2}, P^{2} ; M^{2}\right)\right], \\
\frac{\partial \mathcal{K}_{\mathrm{PS}}^{F F}}{\partial P^{2}}= & -\frac{\mathcal{K}_{\mathrm{PS}}^{F F}}{P^{2}}-8 \frac{\left(M_{l}+M_{h}\right)^{2}}{2 P^{2}}\left\{\left[I_{\log }\left(M^{2}\right)-Z_{0}\left(M_{l}^{2}, M_{h}^{2}, P^{2} ; M^{2}\right)\right]\right. \\
& \left.+\left[P^{2}+\left(M_{l}-M_{h}\right)^{2}\right] Y_{1}\left(M_{l}^{2}, M_{h}^{2}, P^{2} ; M^{2}\right)\right\},
\end{aligned}
$$

where we have defined

$$
\begin{aligned}
Y_{1}\left(M_{l}^{2}, M_{h}^{2}, P^{2} ; M^{2}\right)= & \frac{1}{(4 \pi)^{2}} \int_{0}^{1} d z z(1-z)\left[e^{-H(z) \tau_{\mathrm{ir}}^{2}}-1\right], \\
Y_{2}\left(M_{l}^{2}, M_{h}^{2}, P^{2} ; M^{2}\right)= & \frac{1}{P 2} Z_{1}\left(M_{l}^{2}, M_{h}^{2}, P^{2} ; M^{2}\right) \\
& +\frac{1}{2 P^{2}}\left[Z_{0}\left(M_{l}^{2}, M_{h}^{2}, P^{2} ; M^{2}\right)+\left(P^{2}+M_{h}^{2}-M_{l}^{2}\right) Y_{1}\left(M_{l}^{2}, M_{h}^{2}, P^{2} ; M^{2}\right)\right] .
\end{aligned}
$$

From the eigenvalue equation, one obtains

$$
E_{\mathrm{PS}}^{l h}(P)=\frac{\mathcal{K}_{\mathrm{PS}}^{E F}}{\left(\frac{3 m_{G}^{2}}{4 \pi \alpha_{\mathrm{IR}}}-\mathcal{K}_{\mathrm{PS}}^{E E}\right)} F_{\mathrm{PS}}^{l h}(P) .
$$

Combining this with Eq. (A4), the individual amplitudes $E_{\mathrm{PS}}^{l h}(P)$ and $F_{\mathrm{PS}}^{l h}(P)$ are determined.

\section{Appendix B: Vector kernel}

After performing the necessary number of subtractions in the integrand of Eq. (18), one obtains again finite integrals and divergent integrals that are independent of $\eta_{ \pm}$and symmetry violating terms:

$$
\begin{aligned}
\mathcal{K}_{\mathrm{V}}^{E E}(P)= & \frac{4}{3}\left\{-\left[P^{2}+\left(\Delta M_{l h}\right)^{2}-4 M_{l} M_{h}\right]\left[I_{\log }\left(M^{2}\right)-Z_{0}\left(M_{l}^{2}, M_{h}^{2}, P^{2}, M^{2}\right)\right]\right. \\
& +2\left(P^{2}+M_{h}^{2}-M_{l}^{2}\right)\left[Z_{0}\left(M_{l}^{2}, M_{h}^{2}, P^{2} ; M^{2}\right)-Z_{1}\left(M_{l}^{2}, M_{h}^{2}, P^{2} ; M^{2}\right)-\frac{1}{2} I_{\log }\left(M_{l}^{2}\right)\right] \\
& \left.+3 I_{\text {quad }}\left(M_{l}\right)+I_{\text {quad }}\left(M_{h}\right)+\left(\eta_{+}^{2}+\eta_{-}^{2}\right) A_{\mu \nu}\left(M^{2}\right) P_{\mu} P_{\nu}+\frac{1}{2} \frac{P_{\mu} P_{\nu} D_{\mu \nu}\left(M^{2}\right)}{P^{2}}\right\},
\end{aligned}
$$

where $Z_{1}\left(M_{l}^{2}, M_{h}^{2}, P^{2} ; M^{2}\right)$ is another finite integral that can be related [50] to $Z_{0}$ as

$$
\begin{aligned}
Z_{1}\left(M_{l}^{2}, M_{h}^{2}, P^{2} ; M^{2}\right)= & \frac{1}{(4 \pi)^{2}} \int_{0}^{1} d z z \ln \left[\frac{H(z)}{M^{2}}\right]=\frac{1}{2 P^{2}}\left\{M_{h}^{2}\left[1-\ln \left(\frac{M_{h}^{2}}{M^{2}}\right)\right]-M_{l}^{2}\left[1-\ln \left(\frac{M_{l}^{2}}{M^{2}}\right)\right]\right. \\
& \left.+\left(P^{2}+M_{h}^{2}-M_{l}^{2}\right) Z_{0}\left(M_{l}^{2}, M_{h}^{2}, P^{2} ; M^{2}\right)\right\} .
\end{aligned}
$$

The term $D_{\mu \nu}\left(M^{2}\right)$ is defined in Eq. (A3).

The vector meson decay constant is given by

$$
f_{\mathrm{V}}=\left.\frac{N_{c}}{2} E_{V}^{l h}(P) \mathcal{K}_{\mathrm{V}}^{E E}(P)\right|_{P^{2}=-m_{V}^{2}},
$$


where the BS amplitude is normalized as in the case of pseudoscalar mesons and is is fixed as

$$
\frac{1}{\left(E_{V}^{l h}\right)^{2}}=\left.N_{c} \frac{\partial \mathcal{K}_{V}^{E E}(P)}{\partial P^{2}}\right|_{P^{2}=-m_{V}^{2}} .
$$

[1] A. V. Manohar and M. B. Wise, Heavy quark physics (Cambridge University Press, Cambridge, 2000).

[2] A. A. Petrov and A. E. Blechman, Effective Field Theories (World Scientific, Singapore, 2016).

[3] C. D. Roberts and A. G. Williams, Prog. Part. Nucl. Phys. 33, 477 (1994).

[4] R. Alkofer and L. von Smekal, Phys. Rept. 353, 281 (2001).

[5] I. C. Cloet and C. D. Roberts, Prog. Part. Nucl. Phys. 77, 1 (2014).

[6] G. Eichmann, H. Sanchis-Alepuz, R. Williams, R. Alkofer and C. S. Fischer, Prog. Part. Nucl. Phys. 91, 1 (2016).

[7] D. Binosi, L. Chang, J. Papavassiliou, S. X. Qin and C. D. Roberts, Phys. Rev. D 93, 096010 (2016).

[8] P. Maris and P. C. Tandy, Nucl. Phys. Proc. Suppl. 161, 136 (2006).

[9] T. Nguyen, N. A. Souchlas and P. C. Tandy, AIP Conf. Proc. 1361, 142 (2011)

[10] N. Souchlas, J. Phys. G 37, 115001 (2010).

[11] N. Souchlas and D. Stratakis , Phys. Rev. D 81, 114019 (2010).

[12] A. Bashir, L. Chang, I. C. Cloët, B. El-Bennich, Y. X. Liu, C. D. Roberts and P. C. Tandy, Commun. Theor. Phys. 58, 79 (2012).

[13] M. Gómez-Rocha, T. Hilger and A. Krassnigg, Few Body Syst. 56, 475 (2015).

[14] E. Rojas, B. El-Bennich and J. P. B. C. de Melo, Phys. Rev. D 90, 074025 (2014).

[15] M. Gomez-Rocha, T. Hilger and A. Krassnigg, Phys. Rev. D 92, 054030 (2015).

[16] M. Gómez-Rocha, T. Hilger and A. Krassnigg, Phys. Rev. D 93, 074010 (2016).

[17] T. Hilger, M. Gmez-Rocha, A. Krassnigg and W. Lucha, arXiv:1702.06262 [hep-ph].

[18] R. A. Briceño et al., Chin. Phys. C 40, 042001 (2016).

[19] G. Krein, AIP Conf. Proc. 1701, 020012 (2016).

[20] F. E. Serna, M. A. Brito and G. Krein, AIP Conf. Proc. 1701, 100018 (2016).

[21] Y. Nambu and G. Jona-Lasinio, Phys. Rev. 122, 345 (1961).

[22] U. Vogl and W. Weise, Prog. Part. Nucl. Phys. 27, 195 (1991).

[23] S. Klevansky, Rev. Mod. Phys. 64, 649 (1992).

[24] T. Hatsuda and T. Kunihiro, Phys. Rept. 247, 221 (1994).

[25] J. Bijnens, Phys. Rept. 265, 369 (1996).

[26] L. X. Gutiérrez-Guerrero, A. Bashir, I. C. Cloët and C. D. Roberts, Phys. Rev. C 81, 065202 (2010).

[27] G. Krein, C. D. Roberts and A. G. Williams, Int. J. Mod. Phys. A 7, 5607 (1992).

[28] C. S. Fischer and R. Alkofer, Phys. Rev. D 67, 094020 (2003).

[29] D. Ebert, T. Feldmann and H. Reinhardt, Phys. Lett. B
388, 154 (1996).

[30] H. J. Munczek, Phys. Rev. D 52, 4736 (1995).

[31] A. Bender, C. D. Roberts and L. von Smekal, Phys. Lett. B 380, 7 (1996).

[32] H. L. L. Roberts, C. D. Roberts, A. Bashir, L. X. Gutiérrez-Guerrero and P. C. Tandy, Phys. Rev. C 82, 065202 (2010).

[33] H. L. L. Roberts, A. Bashir, L. X. Gutiérrez-Guerrero, C. D. Roberts and D. J. Wilson, Phys. Rev. C 83, 065206 (2011).

[34] C. Chen, L. Chang, C. D. Roberts, S.-L. Wan and D. J. Wilson, Few Body Syst. 53, 293 (2012).

[35] D. J. Wilson, I. C. Cloët, L. Chang and C. D. Roberts, Phys. Rev. C 85, 025205 (2012).

[36] C. Chen, L. Chang, C. D. Roberts, S. M. Schmidt, S. Wan and D. J. Wilson, Phys. Rev. C 87, 045207 (2013).

[37] K.-L. Wang, Y.-X. Liu, L. Chang, C. D. Roberts and S. M. Schmidt, Phys. Rev. D 87, 074038 (2013).

[38] H. L. L. Roberts, L. Chang, I. C. Cloët and C. D. Roberts, Few Body Syst. 51, 1 (2011).

[39] J. Segovia, C. Chen, C. D. Roberts and S. Wan, Phys. Rev. C 88, 032201 (2013).

[40] J. Segovia, C. Chen, I. C. Cloët, C. D. Roberts, S. M. Schmidt and S. Wan, Few Body Syst. 55, 1 (2014).

[41] Marco A. Bedolla, J. J. Cobos-Martínez, and Adnan Bashir. Phys. Rev. D 92, 054031 (2015). Phys. Rev. D 92, 054031 (2015).

[42] M. A. Bedolla, K. Raya, J. J. Cobos-Martínez and A. Bashir, Phys. Rev. D 93, 094025 (2016).

[43] O. A. Battistel, G. Dallabona and G. Krein, Phys. Rev. D 77 (2008) 065025.

[44] O. A. Battistel and G. Dallabona, Phys. Rev. D 80, 085028 (2009); ibid Phys. Rev. D 94, 085011 (2016).

[45] O. A. Battistel and G. Krein, Mod. Phys. Lett. A 18 (2003) 2255.

[46] R. L. S. Farias, G. Dallabona, G. Krein and O. A. Battistel, Phys. Rev. C 77, 065201 (2008).

[47] R. L. S. Farias, G. Dallabona, G. Krein and O. A. Battistel, Phys. Rev. C 73, 018201 (2006).

[48] R. L. S. Farias, G. Krein, G. Dallabona and O. A. Battistel, Nucl. Phys. A 790, 332 (2007).

[49] R. L. S. Farias, D. C. Duarte, G. Krein and R. O. Ramos, Phys. Rev. D 94, 074011 (2016).

[50] O. A Battistel, PhD Thesis 1999, Universidade Federal de Minas Gerais, Brazil (unpublished).

[51] M. Sampaio, A. P. Baeta Scarpelli, B. Hiller, A. Brizola, M. C. Nemes and S. Gobira, Phys. Rev. D 65, 125023 (2002).

[52] J. C. Collins, Renormalization : An Introduction to Renormalization, The Renormalization Group, and the Operator Product Expansion (Cambridge University Press, Cambridge, 1984).

[53] D. Blaschke, P. Costa, and Yu. L. Kalinovsky, Phys. Rev. 
D 85, 034005 (2012).

[54] B. El-Bennich, G. Krein, E. Rojas and F. E. Serna, Few Body Syst. 57, 955 (2016).

[55] R. Casalbuoni, R. Gatto, G. Nardulli and M. Ruggieri, Phys. Rev. D 68, 034024 (2003)

[56] B. El-Bennich, O. Leitner, J.-P. Dedonder and B. Loiseau, Phys. Rev. D 79, 076004 (2009).

[57] E. O. da Silva, J. P. B. C. de Melo, B. El-Bennich and V. S. Filho, Phys. Rev. C 86, 038202 (2012).

[58] B. El-Bennich, J. P. B. C. de Melo and T. Frederico, Few Body Syst. 54, 1851 (2013).

[59] J. P. B. C. de Melo, K. Tsushima, B. El-Bennich, E. Rojas and T. Frederico, Phys. Rev. C 90, 035201 (2014).

[60] G. H. S. Yabusaki, I. Ahmed, M. A. Paracha, J. P. B. C. de Melo and B. El-Bennich, Phys. Rev. D 92, 034017 (2015).

[61] B. El-Bennich, J. P. B. C. de Melo, B. Loiseau, J.-P. Dedonder and T. Frederico, Braz. J. Phys. 38, 465 (2008).

[62] B. El-Bennich, M. A. Ivanov and C. D. Roberts, Nucl. Phys. Proc. Suppl. 199, 184 (2010).

[63] P. Maris and C. D. Roberts, Phys. Rev. C 56, 3369 (1997).

[64] C. Patrignani et al. (Particle Data Group), Chin. Phys. C 40, 100001 (2016).

[65] P. Maris and P. C. Tandy, Phys. Rev. C 60, 055214 (1999).

[66] D. Becirevic, V. Lubicz, F. Sanfilippo, S. Simula and C. Tarantino, JHEP 1202, 042 (2012).
[67] J. Haidenbauer, G. Krein, U.-G. Meißner and A. Sibirtsev, Eur. Phys. J. A 33, 107 (2007).

[68] J. Haidenbauer, G. Krein, U.-G. Meißner and A. Sibirtsev, Eur. Phys. J. A 37, 55 (2008).

[69] J. Haidenbauer, G. Krein, U.-G. Meißner and L. Tolos, Eur. Phys. J. A 47, 18 (2011).

[70] C. E. Fontoura, G. Krein and V. E. Vizcarra, Phys. Rev. C 87, 025206 (2013).

[71] K. Tsushima, D. H. Lu, A. W. Thomas, K. Saito and R. H. Landau, Phys. Rev. C 59, 2824 (1999).

[72] C. Garcia-Recio, J. Nieves, and L. Tolos, Phys. Lett. B 690, 369 (2010).

[73] C. Garcia-Recio, J. Nieves, L. L. Salcedo, and L. Tolos, Phys. Rev. C 85, 025203 (2012).

[74] S. H. Lee and C. M. Ko, Phys. Rev. C 67, 038202 (2003).

[75] G. Krein, A. W. Thomas and K. Tsushima, Phys. Lett. B 697, 136 (2011).

[76] K. Tsushima, D. H. Lu, G. Krein and A. W. Thomas, Phys. Rev. C 83, 065208 (2011).

[77] G. Krein, PoS ConfinementX , 144 (2012).

[78] B. El-Bennich, G. Krein, L. Chang, C. D. Roberts and D. J. Wilson, Phys. Rev. D 85, 031502 (2012)

[79] G. Krein, EPJ Web Conf. 73, 05001 (2014).

[80] B. El-Bennich, M. A. Paracha, C. D. Roberts and E. Rojas, Phys. Rev. D 95, no. 3, 034037 (2017).

[81] A. Ballon-Bayona, G. Krein and C. Miller, arXiv:1702.08417 [hep-ph].

[82] S. Ghosh, F. E. Serna, B. F. Inchausp, S. K. Das, G. Krein, in preparation. 